\title{
AN OUTBREAK OF STREPTOCOCCAL WOUND SEPSIS: CONTAMINATION OF THE WOUND DURING OPERATION
}

\author{
J. Portnoy, M.D., F.R.C.P.(c), J. Mendelson, M.D., F.R.C.P.(C), J.P. Dechene, M.D., \\ F.R.C.P.(c), AND I. SHRAGOVITCH, M.D., F.R.C.s.(c), F.A.C.S.
}

\section{INTRODUCTION}

Wound INFECTIONS are recognized as potentially serious complications in patients subjected to surgical operations. Outbreaks of post-operative sepsis are not uncommon, and many have been reported in the literature. Such infections add significantly to the severity of the patient's illness and the duration of hospitalization. Accurate information of the incidence, etiology and source of infections acquired within the hospital is essential for prevention.

An outbreak of eight post-operative infections due to group A beta-haemolytic streptococci occurred over a one-month period and was controlled after the implicated source, an anaesthetist was found to be the carrier. The details of the investigation and findings are presented in this report.

\section{Case \#1}

\section{Case Reports}

A 72-year-old man (U-52995) was admitted to the Jewish General Hospital, Montreal, with gastro-intestinal bleeding on September 12, 1973. A gastrotomy and biopsy of a gastric ulcer was performed on September 13. During the first three post-operative days the patient had a temperature of 102 degrees rectally, and a sero-sanguinous discharge from the wound. The temperature spiked to 105 degrees on September 17, and group A beta-haemolytic streptococci were cultured from the wound drainage on September 18. The patient received gentamicin, 120 mg intravenously every eight hours from September 14 to September 22 . No additional antibiotics were administered and the patient was discharged from hospital on September 27, 1973.

\section{Case \#2}

A 42-year-old woman (U-147228) was admitted on September 17, 1973 for evaluation of a right ovarion mass. At laparotomy on September 18, a fibrothecoma of the right ovary was found. A bilateral salpingectomy and right oophorectomy was performed. On September 20, the patient had a rectal temperature of 103 degrees. Ampicillin, $500 \mathrm{mg}$ p.o. every 6 hours was started and continued until her discharge from hospital on September 25. On September 26, she was readmitted to the hospital because of a profuse discharge from her wound. A culture of this material yielded group A beta-haemolytic streptococci. She was treated with erythromycin and was discharged from hospital on October 16, 1973.

From Departments of Medicine and Microbiology, Anaesthesiology and Surgery, Jewish General Hospital, Montreal 249, Quebec, Canada. 


\section{Case \#3}

A 58-year-old man (U-146981) was admitted to the hospital on September 13, 1973 , for investigation of a left rental mass. Marsupialization of a left renal cyst was performed on September 18. Within 24 hours, the patient developed a temperature of 103 degrees and extensive erysipelas extending above and below the incision site. Group A beta-haemolytic streptococci were cultured from the wound. The patient was treated with intravenous penicillin. His subsequent course was one of progressive but slow improvement and he was discharged from hospital on October 15, 1973.

\section{Case \#4}

A 49-year-old lady (O.P.D.-1) was admitted on September 12, 1973, and discharged the same day after having a biopsy of a mass in the right breast. Within 48 hours, she developed a temperature of 101 degrees, chills and erythema of the wound edges. She was seen by her surgeon and was started on tetracycline, 250 $\mathrm{mg}$, every six hours. On September 17, the wound was drained and beta-haemolytic streptococcus group A was cultured from the incision. Tetracycline therapy was continued for a few more days.

\section{Case \#5}

A 47-year-old woman (U-147260) was admitted on September 18, 1973, and bilateral bunionectomies were performed the next day. The patient developed a temperature of 102 degrees, chills and erythema surrounding the incision site on each foot on September 21. Group A beta-haemolytic streptococci were cultured from drainage from the right incision. The patient was treated with intravenous penicillin and was discharged on October 3, 1973.

\section{Case \#6}

A 44-year-old woman (U-147274) was admitted on September 23, 1973, with suspected carcinoma of the breast. A simple mastectomy was performed on September 24. On September 25, she had a temperature of 104 degrees and betahaemolytic streptococci were cultured from her wound, which was drained surgically on September 27. The patient received penicillin from October 6 until her discharge on October 13, 1973.

\section{Case \#7}

A 64-year-old man (U-147577) was admitted on September 24, 1973, for bilateral hernia repair and excision of an epidermoid cyst on the right calf. The operation took place on September 25 and the following day the patient was febrile, with a temperature of 101 degrees. He complained of severe pain in the right calf. The wound on the right calf began to drain spontaneously on September 29 and a culture taken on October 1 showed group A beta-haemolytic streptococci. Both inguinal incisions were red and tender. The patient received cephalexin, $500 \mathrm{mg}$ p.o. six times daily from September 28 to October 2. On October 2, penicillin treatment was started and the wound was drained surgically. The patient was discharged from hospital on October 13, 1973. 


\section{Case \#8}

A 57-year-old man (U-147201) was admitted on September 16, 1973 for investigation of a gastric ulcer. A hemigastrectomy was done on September 26 for adenocarcinoma of the stomach. The following day the patient developed a temperature of 102 degrees. Slight sero-sanguinous discharge was noted from the incision on October 3. The patient was given 2 million units of penicillin intravenously every six hours from September 28 to October 3 and was discharged from the hospital on October 7 . He was readmitted to the hospital the same day because of a dehiscence of the wound and group A beta-hemolytic streptococci were cultured from the incision site at this time. The patient was discharged from hospital on October 19, after being treated with penicillin.

\section{DESCRIPTION OF THE OUTBREAK}

Three post-operative surgical wound infections caused by group A betahaemolytic streptococci were discovered on the surgical service of the Jewish General Hospital in Montreal during a one week period from September 17 to September 23, 1973. Epidemiological investigation revealed that the three cases occurred in patients who had undergone elective operations in the departments of general surgery, urology, and orthopedics. The operations had been done on different days, in different operating rooms, with different surgeons, assistants, and scrub nurses. The anaesthetist was the only person associated with the care of all these patients. A survey of all post-operative patients in the hospital was then undertaken and two additional patients with group A beta-haemolytic streptococcal wound infections were discovered. The same anaesthetist administered the anaesthetics to these patients as well. The charts of 80 patients who had been given an anaesthetic by this same anaesthetist during a preceding three-week period were then reviewed. Three additional streptococcal wound infections were discovered. A total of eight post-operative wound infections during this period were considered to represent an epidemic. All post-operative infections had occurred in patients who had undergone elective operations. The clinical manifestations of the outbreak were characteristic. Within 24 to 48 hours after operation, the patients developed chills, fever, and erythema surrounding the incision site. In one patient (U-146981) the fulminant nature of the infection was made evident by an area of extensive erysipelas extending from the left subcostal incision to the mid-chest above and to the iliac crest below.

The anaesthetist implicated stated that he was well, but had had a "cold" a week previously. However, he denied having had a sore throat. He stated that two of his children had sore throats and were being treated for a possible streptococcal pharyngitis by their paediatrician. Cultures of his nasopharynx, tonsils, and anus produced a growth of group A beta-haemolytic streptococci. He was treated with a course of penicillin and following treatment no further cases of streptococcal post-opcrative wound sepsis occurred. 


\section{Discussion}

A serious epidemic of post-operative sepsis was traced to a single reservoir as the probable source of the infection. The epidemiologic investigation showed that the carrier anaesthetist had a mild unrecognized streptococcal nasopharyngitis. In addition, this anaesthetist often permitted his mask to drop, leaving his nose uncovered for prolonged periods of time. The careless use of a face mask by this physician may have been responsible for the outbreak. A good face mask, worn properly, will have a microbial filtration of about 95 per cent and will remain effective for up to eight hours. ${ }^{1}$ The rapidity (24 hours) with which several patients became febrile and developed extensive erythema surrounding the wound suggests that large numbers of bacteria were deposited into the wound during the operation.

The occurrence of a single case of post-operative beta-haemolytic streptococcal wound infection may herald an outbreak of such infections and should prompt immediate epidemiological investigation. All people having contact with the infected patient before or during the operation should have cultures taken from nose, throat, anus, and any skin lesion that may be present. We do not recommend routine streptococcal screening of hospital personnel in the absence of streptococcal infections. However, it may be advisable to do cultures on all personnel who work in the operating room if they have upper respiratory complaints or skin lesions.

The rapid and early (24-48 hours) development of fever, chills, toxicity and cellulitis of the wound should serve as a clue to a beta-haemolytic streptococcal infection. Immediate Gram staining of smears and culture of the wound should be undertaken and appropriate therapy started immediately, since these infections may be fatal." In none of the patients was the etiology of the wound infection suspected before the culture results became known. It is of interest that in cases \#2, \#4,\#7, and \#8, the patients had received an antibiotic to which the organism is sensitive for several days before the wound culture was taken. Case \#8 had received 2 million units of penicillin intravenously every six hours for five days before the organism was cultured from the wound. This would suggest that penicillin treatment alone is not sufficient to eradicate the streptococcus from the infected site. Indeed, the eight patients showed clinical and bacteriological improvement only after spontaneous or surgical drainage. In all cases, response to therapy was slow and discharge from hospital was delayed for many days.

The present outbreak was controlled by rapid identification, isolation and treatment of infected patients and physician due to prompt action of the hospital Infection Control Committee.

Anaesthetists have been implicated as the source of group A beta-haemolytic streptococcal sepsis in other outbreaks ${ }^{(3,4)}$. This report serves as a reminder to anaesthetists of their potential role in surgical wound sepsis.

\section{SUMMARY}

A limited epidemic of post-operative wound infections caused by group A betahaemolytic streptococcus occurred on the surgical service of the Jewish General 
Hospital in Montreal in September of 1973. An occult respiratory infection in an anaesthetist appears to have been the source of this outbreak, which was promptly curbed by appropriate control measures. This report emphasizes the necessity for stringent enforcement of techniques for the prevention of transmission of infection.

\section{RÉSUMÉ}

En septembre 1973, au Départment de Chirurgie du Jewish General Hospital de Montréal, on a observé une petite manifestation épidémique d'infection de plaies opératoires du type streptocoque A beta-hemolytique.

Après une étude détaillée de ces huit cas, une infection asymtomatique des voies respiratoires d'un anesthésiste est apparue comme le facteur étiologique de cette manifestation. Heureusement, grâce aux mesures appropriées, le tout est entré rapidement dans l'ordre.

Ce travail avait pour double but :

(1) de souligner, une fois de plus, l'importance de techniques opératoires aseptiques;

(2) de mettre en garde tout le personnel de la salle d'opération, et en particulier l'anesthésiste, d'un essaimage éventuel dans les plaies opératoires d'une infection quelconque.

\section{ACKNOWLEDGEMENT}

We wish to thank Miss Margo Robitaille, our Infection Control Nurse, for her aid in the epidemiologic investigation.

\section{REFERENCES}

1. Dineen P. Microbial filtration by surgical masks. Surg. Gynecol. Obstet. 133: 812-814 (1971).

2. Quintiliani, R. \& ENGH, G.A. Overwhelming sepsis associated with group A betahemolytic streptococci, J. Bone Joint Surg. 53-A, 1391-1399 (1971).

3. Schaffner, W., Lefrowitz, L.B. Jr., Goodman, J.S., \& Koenig, M. Hospital outbreak of infection with group A streptococci traced to an asymptomatic carrier. N.E.J.M. 280: 1224-1225 (1969).

4. Jewett, J.F., Reid, D.E., Safon, L.E., \& Easterday, C.L. Childbed fever - a continuing entity. J.A. MA. 206: 344-350 (1968). 